\title{
Stability Analysis of Teleoperation System by State Convergence with Variable Time Delay
}

\author{
Julio C. Tafur, Cecilia García, Rafael Aracil, and Roque Saltaren
}

\section{I.INTRODUCTION}

Teleoperation is a process where the operator has some task done at the remote and/or hazardous environments through coordinated control of two robotic arms and has been an active research topic since Geortz and Thompson's demonstration of their first "local-remote" remote control in 1954 [1], teleoperation systems have been used for a number of different tasks, for example, material handling toxic or harmful, operation in remote environments such as submarine or space and perform tasks that require extreme precision and continue to play an increasingly important role for this type of applications in the future [2].

Stability is an important aspect to build a teleoperation system with a high level of telepresence. Certainly, if a system exhibits unstable or closely unstable behavior, the illusion of the operator to be virtually present at the remote end can be destroyed, in addition to possibly make the task difficult or impossible to execute.

The use of the Internet and other networks of packages switching, such as Internet 2 , impose variable time delays, making already established control schemes to develop solutions to deal with instabilities caused by these varying time delays.

At the same time, instability induced by the time delay, requires that the system be controlled in "open loop", reducing the operator to the technique of "wait and see", [3], [4], [5]. For these situations, the general architecture of teleoperation not applies more. In the control design, this latency imposes a tradeoff between the conflicting requirements of stability and performance with the potential for instability increasing by the level of the performance. Hence, the stability problem for time-delayed systems has received considerable attention in recent years.
The first work dealing with the problem of the delay was published in [6], where the system was operated in open loop, therefore not be observed problems of instability [7]. They conclude from several experiments that most operators took the strategy "move and wait" to correct the effects of the significant delay. In 1966 and later will determine that a time equal to or less than 50 ms delay can destabilize bilateral controllers [7], [8], [9].

The problem is due to the power generation in the communication channel that makes this component of the system is not passive [7]. One way to solve this problem is the addition of damping to the master and the slave to absorb the energy generated in the system. However, this technique does not guarantee stability and cause a poor performance [10], [11]. As an alternative, the bilateral control can be modified so that the communication channel acts as a line without loss of transmission [7].

In [12] the problem of bilateral teleoperation where the model of the operator is not passive is considered. Through the use of a PD control strategy without considering the delay shows that of nonlinear teleoperation system is asymptotically stable. When the delay of the communication channel is considered, for a range of coupling proportional gains, the positions converge asymptotically to a non-zero equilibrium point.

In [13] the feedback interconnection of non-linear systems with finite gain L2 is analyzed. In the case of constant delay shows classic small gain conditions to allow stable closed-loop connection which is delayindependent. In the case of variable delay, to ensure the independence of the stability with the delay, they proposed a small modified gain condition which depends of maximum rate of change of the delay.

In [14] proved that it is possible to achieve a stable behavior of teleoperation system with similar schemes to simple PD algorithms, even without the delayed action of the 
derivative, under the classic assumption of the passivity of the operator.

On the state convergence control technique [15], presents a state space formulation for a linear system of $n$ order, through a control algorithm based on the feedback of position and speed of the manipulators signals, allow the remote manipulator to follow to the local handler through the state convergence even when there is a delay in the communication channel.

The method has been validated experimentally in teleoperator systems with a one degree of freedom [16] and two degrees of freedom [17]. They have carried out studies on adaptive control strategies based on this control scheme [18], designs considering delays in transmission [15], bilateral control by state convergence in teleoperated systems where the structure of the robot master differs from the slave [17].

In [19], we propose a novel control scheme based on state convergence for bilateral teleoperation of $n$ degree-offreedom (DOF) nonlinear robotic systems with constant time delay. In this paper we improved the control scheme [19], analyzing the case of time-varying communication delay.

This new proposal improves the position signal with respect to [15] where signal drift problems arise. The main reason for this improvement is that control strategies are independent of parameter uncertainties in robot models, the human operator and the remote environment.

We demonstrate that the state convergence control scheme can be extended to a non-linear teleoperation system. In addition the strategy can be applied directly to a broad class of common control architectures of teleoperation.

The structure of the paper is, as follows: The mathematical model of the teleoperator system is described in section II. The control scheme and stability analysis is showed in section III. Computer simulations of the proposed control scheme are presented in section IV, while the conclusions are given in section $\mathrm{V}$.

\section{NONLINEAR Model of $n$ DOF TEleOPERATION SySTEM}

Let us consider a teleoperator system where both the local and remote are n-DOF manipulators described by EulerLagrange equations of the form

$$
\begin{aligned}
& \mathbf{M}_{1}\left(\mathbf{q}_{1}\right) \ddot{\mathbf{q}}_{1}+\mathbf{C}_{\mathrm{l}}\left(\mathbf{q}_{1}, \dot{\mathbf{q}}_{1}\right) \dot{\mathbf{q}}_{1}+\mathbf{g}_{1}\left(\mathbf{q}_{1}\right)=\boldsymbol{\tau}_{\mathrm{lc}}+\mathbf{F}_{\mathrm{op}} \\
& \mathbf{M}_{\mathrm{r}}\left(\mathbf{q}_{\mathrm{r}}\right) \ddot{\mathbf{q}}_{\mathrm{r}}+\mathbf{C}_{\mathrm{r}}\left(\mathbf{q}_{\mathrm{r}}, \dot{\mathbf{q}}_{\mathrm{r}}\right) \dot{\mathbf{q}}_{\mathrm{r}}+\mathbf{g}_{\mathrm{r}}\left(\mathbf{q}_{\mathrm{r}}\right)=\boldsymbol{\tau}_{\mathrm{rc}}-\mathbf{F}_{\mathrm{e}}
\end{aligned}
$$

Where $\ddot{\mathbf{q}}_{i}, \dot{\mathbf{q}}_{i}, \mathbf{q}_{i} \in \mathbf{R}^{n}$ represent the acceleration, velocity and position of the joint $\mathrm{i}=\{l, r\}$ where $l$ and $r$ sub-index represent the local and remote manipulator respectively. $\mathbf{M}_{i}\left(\mathbf{q}_{i}\right) \in \mathbf{R}^{n \times n}$ Stands for generalized inertia matrix, $\mathbf{C}_{i}\left(\mathbf{q}_{i}, \dot{\mathbf{q}}_{i}\right) \in \mathbf{R}^{n \times n}$ the Coriolis and centrifugal forces matrix, $\mathbf{g}_{i}\left(\mathbf{q}_{i}\right) \in \mathbf{R}^{n}$ the Gravitational vector, $\boldsymbol{\tau}_{i c} \in \mathbf{R}^{n} \quad$ is the control torques signal, $\mathbf{F}_{\mathrm{h}} \in \mathbf{R}^{n}$ represents the human operator interaction force and, finally, $\mathbf{F}_{\mathrm{e}} \in \mathbf{R}^{n}$ is the environment interaction force.
In the block diagram of the teleoperator system, Fig. 1, the dynamics of the local and remote manipulator are given by (1).

Assumption 1: It is supposed that the interaction of the human operator with the local handle is a constant force in the following way [12]: $\mathbf{F}_{\mathrm{h}}=\mathbf{F}_{\mathrm{op}}$

Assumption 2: The interaction of the environment with the remote manipulator is considered passive: $\mathbf{F}_{\mathrm{e}}=\mathbf{K}_{\mathrm{e}} \mathbf{q}_{\mathrm{r}}+\mathbf{B}_{\mathrm{e}} \dot{\mathbf{q}}_{\mathrm{r}}$, where $\mathbf{K}_{\mathrm{e}}, \mathbf{B}_{\mathrm{e}}$ are definite positive matrix $\in \mathbf{R}^{n \times n}$.

Assumption 3: It is supposed that $T_{1}(t)$ and $T_{\mathrm{r}}(t)$ are continuously differentiable functions, which have an upper bound know $T_{\mathrm{i}}^{+}$defined by: $0 \leq T_{i}(t) \leq T_{i}^{+}<\infty, \quad\left|\dot{T}_{i}(t)\right|<1, \quad i=l, r$

In addition, the bound of round-trip delay communication channel is also known $T_{l r}^{+}=T_{l}^{+}+T_{r}^{+}$.

We proposed the control law (2) as shown in Fig. 1, this control law compensates for gravitational forces [20], so that the control torques $\tau_{i c}$ are given by:

$\boldsymbol{\tau}_{\mathrm{lc}}=\boldsymbol{\tau}_{1}+\mathbf{g}_{1}\left(\mathbf{q}_{1}\right), \quad \boldsymbol{\tau}_{\mathrm{rc}}=\boldsymbol{\tau}_{\mathrm{r}}+\mathbf{g}_{\mathrm{r}}\left(\mathbf{q}_{\mathrm{r}}\right)$

Replacing (2) in (1) yields:

$$
\begin{aligned}
& \mathbf{M}_{1}\left(\mathbf{q}_{1}\right) \ddot{\mathbf{q}}_{1}+\mathbf{C}_{1}\left(\mathbf{q}_{1}, \dot{\mathbf{q}}_{1}\right) \dot{\mathbf{q}}_{1}=\boldsymbol{\tau}_{1}+\mathbf{F}_{\mathrm{op}} \\
& \mathbf{M}_{\mathrm{r}}\left(\mathbf{q}_{\mathrm{r}}\right) \ddot{\mathbf{q}}_{\mathrm{r}}+\mathbf{C}_{\mathrm{r}}\left(\mathbf{q}_{\mathrm{r}}, \dot{\mathbf{q}}_{\mathrm{r}}\right) \dot{\mathbf{q}}_{\mathrm{r}}=\boldsymbol{\tau}_{\mathrm{r}}-\mathbf{F}_{\mathrm{e}}
\end{aligned}
$$

\section{State Convergence Algorithm With Variable TIME DELAY}

Consider a new version of the state convergence algorithm taking in count the time variable delay for nonlinear systems as show in Fig 1. The local and remote manipulator (1) is connected via a communication channel with a variable time delay, $T_{i}(t), i=\{l, r\}$.

Consider the control algorithm for state convergence for the non-linear case, the coupling torque for the local and remote manipulator is given by:

$$
\begin{aligned}
& \boldsymbol{\tau}_{1}=\mathbf{K}_{11} \mathbf{q}_{1}+\mathbf{K}_{12} \dot{\mathbf{q}}_{1}+\mathbf{R}_{11} \mathbf{q}_{\mathrm{r}}\left(t-T_{r}(t)\right)+\mathbf{R}_{12} \dot{\mathbf{q}}_{\mathrm{r}}\left(t-T_{r}(t)\right) \\
& \boldsymbol{\tau}_{\mathrm{r}}=\mathbf{K}_{\mathrm{r} 1} \mathbf{q}_{\mathrm{r}}+\mathbf{K}_{\mathrm{r} 2} \dot{\mathbf{q}}_{\mathrm{r}}+\mathbf{R}_{\mathrm{r} 1} \mathbf{q}_{1}\left(t-T_{l}(t)\right)+\mathbf{R}_{\mathrm{r} 2} \dot{\mathbf{q}}_{1}\left(t-T_{l}(t)\right)+G \mathbf{F}_{\mathrm{op}}\left(t-T_{l}(t)\right)
\end{aligned}
$$

Where: $\mathbf{K}_{11}, \mathbf{K}_{12}, \mathbf{R}_{11}, \mathbf{R}_{12}, \mathbf{K}_{\mathrm{r} 1}, \mathbf{K}_{\mathrm{r} 2}, \mathbf{R}_{\mathrm{r} 1}$ and $\mathbf{R}_{\mathrm{r} 2}$ are order $n \times n$ constants matrices. $G$ is a constant.

From assumption 1, and 2, and (3) and (4), one knows that the equilibrium points of the position of local and remote manipulator defined as $\overline{\mathbf{q}}_{1} \in \mathbf{R}^{n}$ and $\overline{\mathbf{q}}_{\mathrm{r}} \in \mathbf{R}^{n}$, satisfy (5).

$$
\begin{aligned}
& \mathbf{0}=\mathbf{K}_{\mathrm{ll}} \overline{\mathbf{q}}_{\mathrm{l}}+\mathbf{R}_{\mathrm{ll}} \overline{\mathbf{q}}_{\mathrm{r}}+\overline{\mathbf{F}}_{\mathrm{op}} \\
& \mathbf{0}=\mathbf{K}_{\mathrm{r} 1} \overline{\mathbf{q}}_{\mathrm{r}}+\mathbf{R}_{\mathrm{r} 1} \overline{\mathbf{q}}_{\mathrm{l}}+G \overline{\mathbf{F}}_{\mathrm{op}}\left(t-T_{l}(t)\right)-\mathbf{K}_{\mathrm{e}} \overline{\mathbf{q}}_{\mathrm{r}}
\end{aligned}
$$

In order to simplify the mathematical demonstration, it is convenient to settle the origin point, that is $[\mathbf{0}] \in \mathbf{R}^{n}$, like the equilibrium of the system. Applying a coordinate transformation like this: 


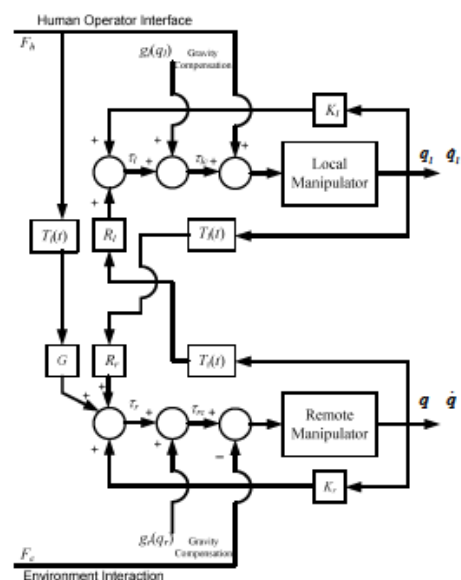

Figure 1. Block diagram of nonlinear control of teleoperation system considering delay.

$$
\begin{aligned}
& \widetilde{\mathbf{q}}_{1}(t)=\mathbf{q}_{1}(t)-\overline{\mathbf{q}}_{1} \rightarrow \mathbf{q}_{1}(t)=\widetilde{\mathbf{q}}_{1}+\overline{\mathbf{q}}_{1} \\
& \widetilde{\mathbf{q}}_{\mathbf{I}}(t)=\mathbf{q}_{\mathbf{I}}(t)-\overline{\mathbf{q}}_{\mathrm{I}} \rightarrow \mathbf{q}_{\mathbf{I}}(t)=\widetilde{\mathbf{q}}_{\mathbf{I}}+\overline{\mathbf{q}}_{\mathrm{I}}
\end{aligned}
$$

In the new variables $\widetilde{\mathbf{q}}_{1}(t), \widetilde{\mathbf{q}}_{\mathrm{r}}(t)$, the system has equilibrium at the origin.

Replacing (6), (7) and (4) in (3) the dynamics of a bilateral teleoperation system in closed-loop is given by:

$\mathbf{M}_{1} \ddot{\tilde{\mathbf{q}}}_{1}+\mathbf{C}_{1} \dot{\tilde{\mathbf{q}}}_{1}=\mathbf{K}_{\mathrm{l}} \tilde{\mathbf{q}}_{1}+\mathbf{R}_{\mathrm{l1}} \tilde{\mathbf{q}}_{r}\left(t-T_{r}(t)\right)+\mathbf{K}_{\mathrm{r}} \dot{\tilde{\mathbf{q}}}_{1}+\mathbf{R}_{\mathrm{l}} \dot{\tilde{\mathbf{q}}}_{r}\left(t-T_{r}(t)\right)$

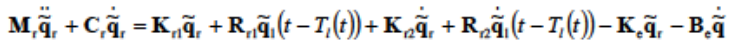

The following theorem describes stability properties concerned with stability of equilibrium points of the closed loop teleoperation (8) with control algorithm given by (4).

\section{Theorem 2.1:}

For the bilateral teleoperation system given by (8), setting the control gains as

$$
\begin{array}{llll}
\mathbf{K}_{11}=-\mathbf{K}, & \mathbf{K}_{\mathrm{r} 2}=-\left(2 \mathbf{K}_{1}+\mathbf{K}_{\mathrm{rd}}\right), & \mathbf{K}_{\mathrm{rl}}=-\mathbf{K}, & \mathbf{R}_{\mathrm{r} 2}=2 \mathbf{K}_{\mathrm{ld}} \\
\mathbf{R}_{\mathrm{ll}}=\mathbf{K}, & \mathbf{K}_{\mathrm{r} 2}=-\left(2 \mathbf{K}_{1}+\mathbf{K}_{\mathrm{rd}}\right), & \mathbf{R}_{\mathrm{r} 1}=\mathbf{K}, & \mathbf{R}_{\mathrm{r} 2}=2 \mathbf{K}_{\mathrm{rd}}
\end{array}
$$

Where: $\mathbf{K}_{1}$ and $\mathbf{K}$ are positive definite constant diagonal matrices. by

$\mathbf{K}_{\mathrm{ld}}$ and $\mathbf{K}_{\mathrm{rd}}$ are positive definite diagonal matrices given

$$
\mathbf{K}_{\mathrm{ld}}=\left(1-\dot{T}_{r}(t)\right) \mathbf{K}_{1} \quad, \quad \mathbf{K}_{\mathrm{rd}}=\left(1-\dot{T}_{l}(t)\right) \mathbf{K}_{1}
$$

If the following is satisfied:

$$
\mathbf{K}_{1}-\frac{\alpha_{1}}{2} \mathbf{K}-\frac{T_{l}^{+^{2}}}{2 \alpha_{2}} \mathbf{K}>\mathbf{0}, \quad \mathbf{K}_{1}-\frac{\alpha_{2}}{2} \mathbf{K}-\frac{T_{r}^{+^{2}}}{2 \alpha_{1}} \mathbf{K}>\mathbf{0}
$$

Where $\alpha_{1}, \alpha_{2}$ and time delay $T_{\mathrm{i}}^{+}$for $i=l, r$ are scalar constants, then the equilibrium point at the origin is asymptotically stable. $\lim _{\mathrm{t} \rightarrow \infty} \widetilde{\mathbf{q}}_{1}=\lim _{t \rightarrow \infty} \widetilde{\mathbf{q}}_{\mathrm{I}}=\lim _{t \rightarrow \infty} \dot{\tilde{\mathbf{q}}}_{1}=\lim _{t \rightarrow \infty} \dot{\tilde{\mathbf{q}}}_{\mathrm{r}}=0$

\section{Proof:}

For the stability analysis considering the constant delay, we used a Lyapunov-Krasovskii functional [21], [22], [23].

Defining $V$, a positive definite function, as:

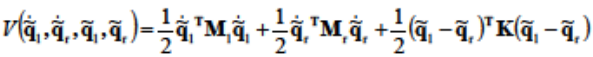

$$
\begin{aligned}
& +\frac{1}{2} \tilde{\mathbf{q}}_{t}^{\mathrm{T}} \mathbf{K}_{\mathrm{e}} \tilde{\mathbf{q}}_{t}+\int_{t-T, t(t)}^{t} \dot{\tilde{\mathbf{q}}}^{\mathrm{T}}(\xi) \mathbf{K}_{1} \dot{\tilde{\mathbf{q}}}_{\mathrm{i}}(\xi) d \xi+\int_{t-T(t)}^{t} \dot{\tilde{\mathbf{q}}}_{t}^{\mathrm{T}}(\xi) \mathbf{K}_{1} \dot{\tilde{\mathbf{q}}}_{r}(\xi) d \xi
\end{aligned}
$$

Where $T_{\mathrm{i}}(t)$ for $i=l, r$ is the variable delay of communication channel and $\mathbf{K}, \mathbf{K}_{\mathrm{e}}$ y $\mathbf{K}_{1}$, positive definite constant diagonal matrices.

The time derivative of the above Lyapunov function along the system trajectories described by (8) is given by:

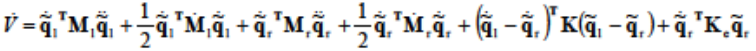

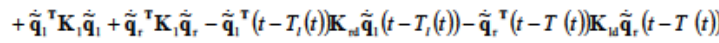

By replacing (8), simplifying and grouping terms, using (9) and the property of the robots dynamics [20], it yields,

$$
\begin{aligned}
& \dot{V}=\dot{\tilde{\mathbf{q}}}_{1}{ }^{\mathrm{T}}(t) \mathbf{K}\left[\widetilde{\mathbf{q}}_{t}(t-T(t))-\widetilde{\mathbf{q}}_{t}(t)\right]-\left[\dot{\tilde{q}}_{t}(t-T(t))-\dot{\tilde{\mathbf{q}}}_{1}(t)\right] \mathbf{K}_{\mathrm{u}}^{\mathrm{T}} \mathbf{K}_{\mathrm{q}}\left[\dot{\tilde{\mathbf{q}}}_{t}(t-T(t))-\dot{\tilde{\mathbf{q}}}_{1}(t)\right]
\end{aligned}
$$

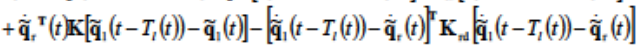

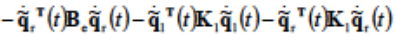

Define

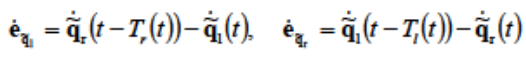

We can rewrite (14) as follows:

$$
\begin{aligned}
& \dot{V}=\dot{\tilde{\mathbf{q}}}_{1} \mathbf{K}\left[\tilde{\mathbf{q}}_{\mathrm{r}}\left(t-T_{r}(t)\right)-\tilde{\mathbf{q}}_{\mathrm{r}}(t)\right]-\dot{\mathbf{e}}_{\tilde{q}_{1}}^{\mathrm{T}} \mathbf{K}_{1 \mathrm{~d}} \dot{\mathbf{e}}_{\boldsymbol{q}_{1}}+\dot{\tilde{\mathbf{q}}}_{\mathrm{r}}{ }^{\mathrm{T}} \mathbf{K}\left[\tilde{\mathbf{q}}_{1}\left(t-T_{l}(t)\right)-\tilde{\mathbf{q}}_{1}(t)\right] \\
& -\dot{\mathbf{e}}_{\tilde{\mathrm{q}}}^{\mathrm{T}} \mathbf{K}_{\mathrm{rd}} \dot{\mathbf{e}}_{\tilde{\mathrm{q}}_{\mathrm{z}}}-\dot{\tilde{\mathbf{q}}}_{\mathrm{r}}^{\mathrm{T}} \mathbf{B}_{\mathrm{o}} \dot{\tilde{\mathbf{q}}}_{\mathrm{r}}-\dot{\tilde{\mathbf{q}}}_{1}^{\mathrm{T}} \mathbf{K}_{1} \dot{\tilde{\mathbf{q}}}_{1}-\dot{\tilde{\mathbf{q}}}_{\mathrm{r}}{ }^{\mathrm{T}} \mathbf{K}_{1} \dot{\tilde{\mathbf{q}}}_{\mathrm{r}}
\end{aligned}
$$

Taking into account that: $\tilde{\mathbf{q}}_{\mathrm{i}}\left(t-T_{i}(t)\right)-\tilde{\mathbf{q}}_{\mathrm{i}}(t)=-\int_{0}^{T_{i}(t)} \tilde{\widetilde{\mathbf{q}}}_{\mathrm{i}}(t-\sigma) d \sigma$

And by replacing in (16), the following result can be obtained:

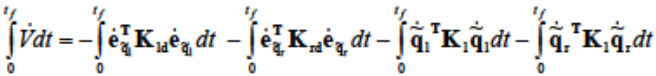

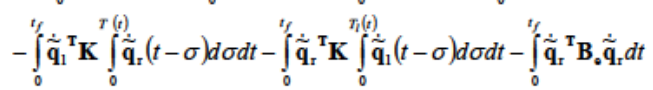

For any vector signals $\mathbf{x}, \mathbf{y} \in \mathbf{R}^{\mathbf{n}}$, for a definite positive diagonal matrix $\mathbf{K} \in \mathbf{R}^{n \times n}, \alpha \in \mathbf{R}^{+}$and for any continuously differentiable functions, which have an upper bound know $T_{\mathrm{i}}^{+}$ defined by $T_{i}(t) \leq T_{i}^{+}<\infty$, we have that:

$$
-2 \int_{0}^{t_{f}} \dot{\mathbf{x}}^{\mathrm{T}} \mathbf{K} \int_{0}^{T_{i}(t)} \dot{\mathbf{y}}(\mathbf{t}-\sigma) d \sigma d t \leq \alpha \int_{0}^{t_{f}} \dot{\mathbf{x}}^{\mathrm{T}} \mathbf{K} \dot{\mathbf{x}} d t+\frac{T_{i}{ }^{2}}{\alpha} \int_{0}^{t_{f}} \dot{\mathbf{y}}^{\mathrm{T}} \mathbf{K} \dot{\mathbf{y}} d t
$$

By replacing (18) into (17)

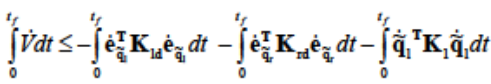

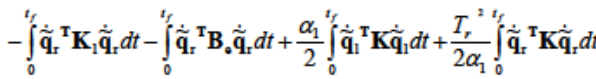

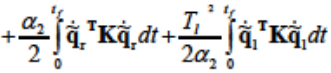

Therefore, integral inequality (19) is reduced to; 


$$
\begin{aligned}
\int_{0}^{t_{f}} \dot{V} d t & \leq-\mu\left(\mathbf{K}_{\mathrm{ld}}\right)\left\|\dot{\boldsymbol{|}}_{\tilde{\mathrm{q}}_{1}}\right\|_{2}^{2}-\mu\left(\mathbf{K}_{\mathrm{rd}}\right)\left\|\dot{\mathbf{e}}_{\tilde{\mathrm{q}}_{\mathrm{r}}}\right\|_{2}^{2}-\mu\left(\mathbf{B}_{\mathrm{c}}\right)\left\|\dot{\tilde{\mathbf{q}}}_{\mathrm{r}}\right\|_{2}^{2} \\
& -\mu\left(\mathbf{K}_{1}-\frac{\alpha_{1}}{2} \mathbf{K}-\frac{T_{l}^{2}}{2 \alpha_{2}} \mathbf{K}\right)\left\|\dot{\mathbf{q}}_{1}\right\|_{2}^{2} \\
& -\mu\left(\mathbf{K}_{1}-\frac{\alpha_{2}}{2} \mathbf{K}-\frac{T_{r}^{2}}{2 \alpha_{1}} \mathbf{K}\right)\left\|\dot{\tilde{\mathbf{q}}}_{\mathrm{r}}\right\|_{2}^{2}
\end{aligned}
$$

Where $\mu(\mathbf{A})$ specifies the smallest eigenvalue of $\mathbf{A}$, and the notation $\|\cdot\|_{2}$ specifies $L_{2}$ norm of a signal in the interval $\left[0, t_{f}\right\rfloor$.

Because $\mathbf{B}_{\mathrm{e}}, \mathbf{K}_{\mathrm{ld}}$ and $\mathbf{K}_{\mathrm{rd}}$ are definite positive matrix, from (20), $\int_{0}^{t_{f}} \dot{V} d t \leq 0$ if the relations in (21) are satisfied:

$$
\mathbf{K}_{1}-\frac{\alpha_{1}}{2} \mathbf{K}-\frac{T_{l}{ }^{2}}{2 \alpha_{2}} \mathbf{K}>0, \quad \mathbf{K}_{1}-\frac{\alpha_{2}}{2} \mathbf{K}-\frac{T_{r}{ }^{2}}{2 \alpha_{1}} \mathbf{K}>0
$$

Considering $\lim _{t_{f} \rightarrow \infty} \int_{0}^{\infty} \dot{V} d t \leq 0$, we conclude that the signals $\left\{\dot{\tilde{\mathbf{q}}}_{1}, \dot{\widetilde{\mathbf{q}}}_{\mathrm{r}}, \widetilde{\mathbf{q}}_{\mathrm{r}}-\widetilde{\mathbf{q}}_{1}, \widetilde{\mathbf{q}}_{\mathrm{r}}\right\} \in L_{\infty}$ and $\left\{\dot{\widetilde{\mathbf{q}}}_{1}, \dot{\widetilde{\mathbf{q}}}_{\mathrm{r}}, \dot{\mathbf{e}}_{\widetilde{\mathrm{q}}_{1}}, \dot{\mathbf{e}}_{\widetilde{\mathrm{q}}_{\mathrm{r}}}\right\} \in L_{2}$.

Rewritten (8) as:

$$
\begin{aligned}
& \ddot{\widetilde{\mathbf{q}}}_{1}=-\mathbf{M}_{l}^{-1}\left[\mathbf{C}_{1} \dot{\widetilde{\mathbf{q}}}_{1}-\mathbf{K}_{11} \widetilde{\mathbf{q}}_{1}-\mathbf{R}_{11} \widetilde{\mathbf{q}}_{\mathrm{r}}\left(t-T_{r}(t)\right)-\mathbf{K}_{12} \dot{\widetilde{\mathbf{q}}}_{1}-\mathbf{R}_{12} \dot{\widetilde{\mathbf{q}}}_{\mathrm{r}}\left(t-T_{r}(t)\right)\right] \\
& \ddot{\tilde{\mathbf{q}}}_{\mathrm{r}}=-\mathbf{M}_{r}^{-1}\left[\mathbf{C}_{\mathrm{r}} \dot{\tilde{\mathbf{q}}}_{\mathrm{r}}-\mathbf{K}_{\mathrm{r} \mathbf{1}} \widetilde{\mathbf{q}}_{\mathrm{r}}-\mathbf{R}_{\mathrm{r} 1} \widetilde{\mathbf{q}}_{\mathrm{l}}\left(t-T_{l}(t)\right)-\mathbf{K}_{\mathrm{r} 2} \dot{\tilde{\mathbf{q}}}_{\mathrm{r}}-\mathbf{R}_{\mathrm{r} \mathrm{r}} \dot{\tilde{\mathbf{q}}}_{\mathrm{l}}\left(t-T_{l}(t)\right)+\mathbf{K}_{\mathrm{e}} \widetilde{\mathbf{q}}_{\mathrm{r}}+\mathbf{B}_{\mathrm{e}} \dot{\tilde{\mathbf{q}}}_{\mathrm{r}}\right]
\end{aligned}
$$

Rewritten $\widetilde{\mathbf{q}}_{1}-\widetilde{\mathbf{q}}_{\mathrm{r}}\left(t-T_{r}(t)\right)$ as:

$$
\widetilde{\mathbf{q}}_{1}-\widetilde{\mathbf{q}}_{\mathrm{r}}\left(t-T_{r}(t)\right)=\widetilde{\mathbf{q}}_{\mathrm{l}}-\widetilde{\mathbf{q}}_{\mathrm{r}}+\widetilde{\mathbf{q}}_{\mathrm{r}}-\widetilde{\mathbf{q}}_{\mathrm{r}}\left(t-T_{r}(t)\right)
$$

It is known that $\widetilde{\mathbf{q}}_{1}-\widetilde{\mathbf{q}}_{\mathrm{r}} \in L_{\infty}$, therefore will look at $\widetilde{\mathbf{q}}_{\mathrm{r}}-\widetilde{\mathbf{q}}_{\mathrm{r}}\left(t-T_{r}(t)\right)$.

Using the Schwartz's inequality, we have the fact that

$$
\widetilde{\mathbf{q}}_{\mathrm{r}}-\widetilde{\mathbf{q}}_{\mathrm{r}}\left(t-T_{r}(t)\right)=\int_{0}^{T(t)} \dot{\tilde{\mathbf{q}}}_{\mathrm{r}}(t-\sigma) d \sigma \leq T_{r}^{1 / 2}\left\|\dot{\tilde{\mathbf{q}}}_{\mathrm{r}}\right\|_{2} \in L_{\infty}
$$

Using (24) and $\widetilde{\mathbf{q}}_{1}-\widetilde{\mathbf{q}}_{\mathrm{r}} \in L_{\infty}$ in (23) we conclude that $\widetilde{\mathbf{q}}_{1}-\widetilde{\mathbf{q}}_{\mathrm{r}}\left(t-T_{r}(t)\right) \in L_{\infty}$. In the same manner we can prove that $\widetilde{\mathbf{q}}_{\mathrm{r}}-\widetilde{\mathbf{q}}_{1}\left(t-T_{l}(t)\right) \in L_{\infty}$.

From (22), taking into account the basic properties of the dynamic model for robots [20], the fact that $\left\{\dot{\tilde{\mathbf{q}}}_{1}, \dot{\widetilde{\mathbf{q}}}_{\mathrm{r}}, \widetilde{\mathbf{q}}_{1}-\widetilde{\mathbf{q}}_{\mathrm{r}}, \widetilde{\mathbf{q}}_{\mathrm{r}}-\widetilde{\mathbf{q}}_{1}\left(t-T_{l}(t)\right), \widetilde{\mathbf{q}}_{1}-\widetilde{\mathbf{q}}_{\mathrm{r}}\left(t-T_{r}(t)\right)\right\} \in L_{\infty}$, ensure that $\left\{\ddot{\widetilde{q}}_{1}, \ddot{\mathbf{q}}_{\mathrm{r}}\right\} \in L_{\infty}$ are uniformly continuous [24]. Also as $\left\{\dot{\widetilde{\mathbf{q}}}_{1}, \dot{\widetilde{\mathbf{q}}}_{\mathrm{r}}\right\} \in L_{2}$, using Barbalat's Lemma [25], it can be concluded that:

$$
\lim _{t \rightarrow \infty} \dot{\widetilde{\mathbf{q}}}_{1}=\lim _{t \rightarrow \infty} \dot{\widetilde{\mathbf{q}}}_{\mathrm{r}}=\lim _{t \rightarrow \infty} \dot{\mathbf{\mathrm { q }}}_{\widetilde{\mathrm{q}}_{\mathrm{I}}}=\lim _{t \rightarrow \infty} \dot{\mathbf{\mathrm { q }}}_{\widetilde{\mathrm{q}}_{\mathrm{r}}}=\mathbf{0}
$$

The signal continuity implies that the integral exists and this bounded by:

$$
\int_{0}^{\infty} \ddot{\widetilde{\mathbf{q}}}_{\mathrm{i}}(\sigma) d \sigma=\dot{\tilde{\mathbf{q}}}_{\mathrm{i}}(\sigma)-\dot{\widetilde{\mathbf{q}}}_{\mathrm{i}}(0)
$$

Evaluating the limit as $t \rightarrow \infty$ and using the fact that $\left|\dot{\tilde{\mathbf{q}}}_{\mathrm{i}}\right| \rightarrow 0$ yields

$\int_{0}^{\infty} \ddot{\widetilde{\mathbf{q}}}_{\mathrm{i}}(\sigma) d \sigma=-\dot{\widetilde{\mathbf{q}}}_{\mathrm{i}}(0)$

This is bounded.

As shown above $\lim _{t \rightarrow \infty} \dot{\widetilde{\mathbf{q}}}_{1}=\lim _{t \rightarrow \infty} \dot{\widetilde{\mathbf{q}}}_{\mathrm{r}}=\mathbf{0} . \quad$ Using Barbalat's Lemma [26] we prove that $\lim _{t \rightarrow \infty} \ddot{\widetilde{\mathbf{q}}}_{1}=\lim _{t \rightarrow \infty} \ddot{\widetilde{\mathbf{q}}}_{\mathrm{r}}=0$

The fact that zero convergence of velocities and acceleration, as a result for the dynamics of the system (8) with $\mathbf{K}_{11}=-\mathbf{K}, \quad \mathbf{K}_{\mathrm{r} 1}=-\mathbf{K}, \quad \mathbf{R}_{11}=\mathbf{K}, \quad \mathbf{R}_{\mathrm{r} 1}=\mathbf{K}$ we have that

$$
\begin{aligned}
& \lim _{t \rightarrow \infty}\left\|\widetilde{\mathbf{q}}_{\mathrm{r}}\left(t-T_{r}(t)\right)-\widetilde{\mathbf{q}}_{\mathrm{l}}\right\|=\mathbf{0} \\
& \lim _{t \rightarrow \infty}\left\|\widetilde{\mathbf{q}}_{\mathrm{l}}\left(t-T_{l}(t)\right)-\widetilde{\mathbf{q}}_{\mathrm{r}}\right\|=\mathbf{K}^{-1} \mathbf{K}_{\mathrm{e}} \lim _{t \rightarrow \infty} \widetilde{\mathbf{q}}_{\mathrm{r}}
\end{aligned}
$$

Taking into account

$$
\begin{aligned}
& \widetilde{\mathbf{q}}_{\mathbf{1}}\left(t-T_{l}(t)\right)=\widetilde{\mathbf{q}}_{\mathbf{l}}(t)-\int_{t-T_{l}(t)}^{t} \dot{\mathbf{q}}_{1}(t) d t \quad, \quad \widetilde{\mathbf{q}}_{\mathrm{r}}\left(t-T_{r}(t)\right)=\widetilde{\mathbf{q}}_{\mathrm{r}}(t)-\int_{t-T(t)}^{t} \dot{\tilde{\mathbf{q}}}_{\mathrm{r}}(t) d t \\
& \lim _{t \rightarrow \infty} \dot{\widetilde{\mathbf{q}}}_{1}=\lim _{t \rightarrow \infty} \dot{\widetilde{\mathbf{q}}}_{\mathrm{r}}=\mathbf{0}
\end{aligned}
$$

it yields:

$\lim _{t \rightarrow \infty}\left\|\widetilde{\mathbf{q}}_{\mathrm{r}}(t)-\widetilde{\mathbf{q}}_{1}(t)\right\|=\mathbf{0} \quad, \lim _{t \rightarrow \infty}\left\|\widetilde{\mathbf{q}}_{1}(t)-\widetilde{\mathbf{q}}_{\mathrm{r}}(t)\right\|=\mathbf{K}^{-1} \mathbf{K}_{\mathrm{e}} \lim _{t \rightarrow \infty} \widetilde{\mathbf{q}}_{\mathrm{r}}(t){ }_{(26)}$

The above equations imply that $\lim _{t \rightarrow \infty} \widetilde{\mathbf{q}}_{1}(t)=\lim _{t \rightarrow \infty} \widetilde{\mathbf{q}}_{\mathbf{r}}(t)=\mathbf{0}$.

Therefore the origin of the system $\left\{\dot{\widetilde{\mathbf{q}}}_{1}, \dot{\widetilde{\mathbf{q}}}_{\mathrm{r}}, \widetilde{\mathbf{q}}_{1}, \widetilde{\mathbf{q}}_{\mathrm{r}}\right\}$ is asymptotically stable and $\lim _{t \rightarrow \infty} \mathbf{q}_{1}(t)=\overline{\mathbf{q}}_{1}, \lim _{t \rightarrow \infty} \mathbf{q}_{\mathrm{r}}(t)=\overline{\mathbf{q}}_{\mathrm{r}}$

This guarantees the stability of the teleoperation system.

\section{Simulation}

The dynamics of the teleoperation system have been simulated using Simulink ${ }^{\circledR}$. For a local manipulator, we will use a PHANTOM Omni ${ }^{\circledR}$ haptic device. For a remote manipulator we employed a planar serial arm with three degrees of freedom, actuated by DC motors, [19], [27]:

$\mathbf{M}_{1}\left(\mathbf{q}_{1}\right) \ddot{\mathbf{q}}_{1}+\mathbf{C}_{1}\left(\mathbf{q}_{1}, \dot{\mathbf{q}}_{1}\right) \dot{\mathbf{q}}_{1}+\mathbf{g}_{1}\left(\mathbf{q}_{1}\right)+\mathbf{f}_{1}\left(\dot{\mathbf{q}}_{1}\right)=\boldsymbol{\tau}_{\mathrm{lc}}+\mathbf{F}_{\mathrm{op}}$

$\mathbf{M}_{\mathrm{r}}\left(\mathbf{q}_{\mathrm{r}}\right) \ddot{\mathbf{q}}_{\mathrm{r}}+\mathbf{C}_{\mathrm{r}}\left(\mathbf{q}_{\mathrm{r}}, \dot{\mathbf{q}}_{\mathrm{r}}\right) \dot{\mathbf{q}}_{\mathrm{r}}+\mathbf{g}_{\mathrm{r}}\left(\mathbf{q}_{\mathrm{r}}\right)+\mathbf{f}_{\mathrm{r}}\left(\dot{\mathbf{q}}_{\mathrm{r}}\right)=\boldsymbol{\tau}_{\mathrm{rc}}-\mathbf{F}_{\mathrm{e}}$ [20]:

$\mathbf{f}(\dot{\mathbf{q}}) \in \mathbf{R}^{n}$ is a static model of joints friction, defined by

The controller is designed when the upper bound of the first derivative of the delay is known.

Assume the time delay in both directions is equal $_{r}^{+}=T_{l}^{+}=0.45 \mathrm{sec}$. Fig. 2 shows the time delay used in simulation. Many authors [28], [29] shown how measure these upper bounds on the time-varying delay magnitude $T_{\mathrm{i}}^{+}$ and its variation $\left|\dot{T}_{i}(t)\right|$ over real Internet conditions. 


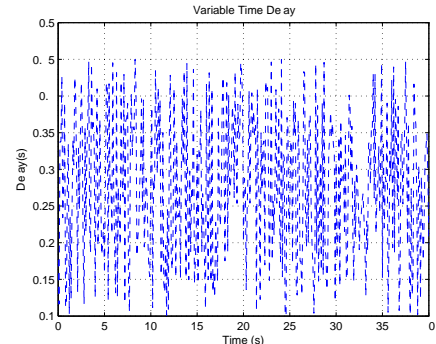

Figure 2. The variable time delay ranges from 0 to $40 \mathrm{sec}$.

The force (torque) applied by the human operator to the joints of the local manipulator to move the remote manipulator is show in Fig. 3.

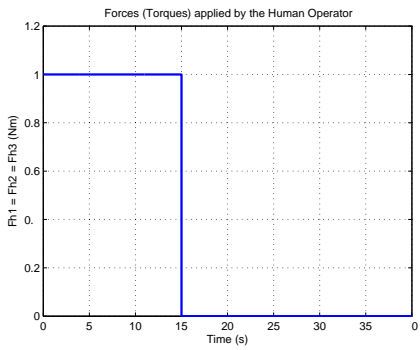

Figure 3. Force, $[\mathrm{Nm}]$ applied by the human operator.

a)

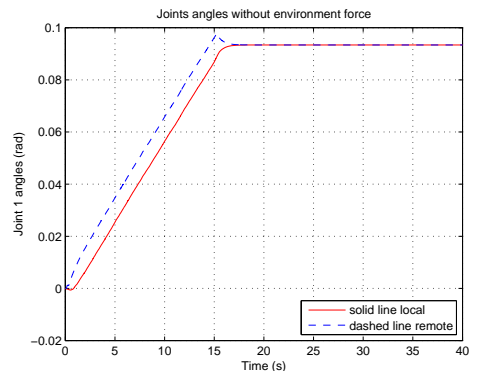

b)

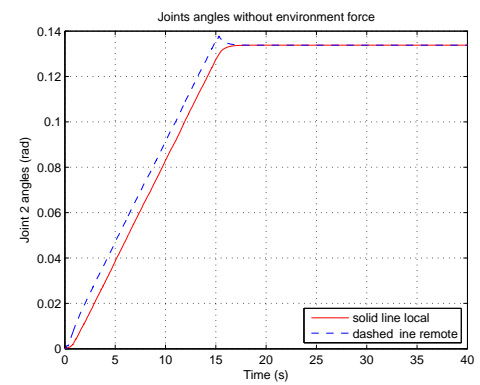

Figure 4. Angular position of local and remote manipulator: a) Joint 1; b) Joint 2.

Letting $\alpha_{1}=T_{r}^{+}, \alpha_{2}=T_{l}^{+}$and $\left|\dot{T}_{i}(t)\right|=\dot{T}_{\max }=0.8$, the gains $\mathbf{K}$ and $\mathbf{K}_{1}$ are calculated using relation (11) as:

$$
\mathbf{K}_{1}=\left[\begin{array}{ccc}
40 & 0 & 0 \\
0 & 30 & 0 \\
0 & 0 & 10
\end{array}\right], \quad \mathbf{K}=\left[\begin{array}{ccc}
80 & 0 & 0 \\
0 & 60 & 0 \\
0 & 0 & 20
\end{array}\right]
$$

Then, the controller's gains values parameters $\mathbf{K}_{11}, \mathbf{K}_{12}$, $\mathbf{K}_{\mathrm{r} 1}, \mathbf{K}_{\mathrm{r} 2}, \mathbf{R}_{11}, \mathbf{R}_{12}, \mathbf{R}_{\mathrm{r} 1}, \mathbf{R}_{\mathrm{r} 2}, \mathbf{K}_{\mathrm{ld}}$ and $\mathbf{K}_{\mathrm{rd}}$ are determined by (9) and (10), in addition $G=1$.

Simulations have been carried out considering two cases: the remote manipulator does not interact with the environment and the remote manipulator interacts with the environment. Their objective is to show that the original controllers proposed on this work do provide position tracking.

a)

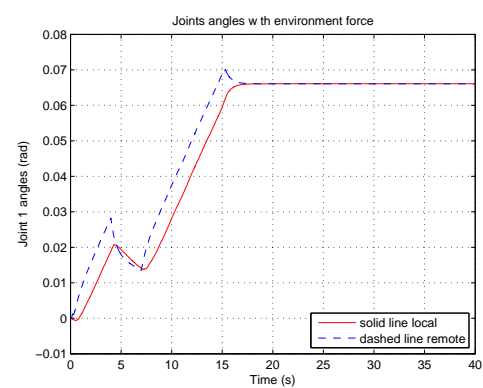

b)

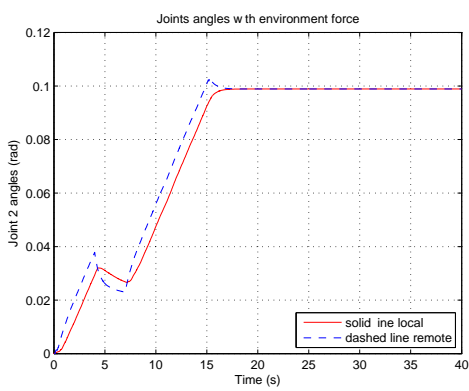

c)

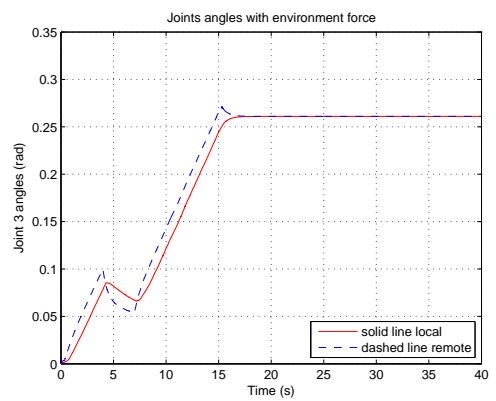

Figure 5. Angular position of local and remote manipulator: a) Joint $1 ;$ b) Joint 2; c) Joint 3.

\section{A. Case A: Without Environment Interaction}

As seen from the simulation waveforms in Fig. 4 for the case when the remote manipulator does not interact with the environment, i.e. interaction force is zero; better tracking performance can be obtained by using the proposed control scheme. The joint angles of the remote manipulator (dashed line) accurately track those of the local manipulator (solid line). When the operator force is zero, at $t=15 \mathrm{sec}$, the position coordination error $\tilde{\mathbf{q}}(t)=\mathbf{q}_{1}(t)-\mathbf{q}_{\mathrm{r}}(t)$ tends to zero and the equilibrium points of the position of local and remote manipulator $\overline{\mathbf{q}}_{1}$ and $\overline{\mathbf{q}}_{\mathrm{r}}$ are identical. The stability of 
teleoperator in closed loop with the controllers of this scheme (4) has been established in Theorem 2.1. The controller guarantees a stable behavior under time delays, and also ensures position tracking.

\section{B. Case B: Environment Interaction}

In order to assess the stability of the contact in simulations, we considered a soft environment modeled by means of a spring -damper system, with gains as: $\mathbf{K}_{\mathrm{c}}=\left[\begin{array}{ccc}25 & 0 & 0 \\ 0 & 25 & 0 \\ 0 & 0 & 25\end{array}\right] \mathrm{N} / \mathrm{m}, \quad \mathbf{B}_{\mathrm{c}}=\left[\begin{array}{lll}1 & 0 & 0 \\ 0 & 1 & 0 \\ 0 & 0 & 1\end{array}\right] \mathrm{N} \cdot \mathrm{s} / \mathrm{m}$

Fig. 5 shows the joints positions of the local and remote manipulator. When the remote manipulator does not contact with the environment $(0-4 \mathrm{~s}$ and $10-40 \mathrm{~s})$ position coordination of the local and remote manipulator position is achieved. Simulations cannot replicate the quality of the human perception, but they provide useful indications about performance of the controller.

\section{CONCLUSION}

In this paper, we propose a novel control scheme that guarantees the stability of the overall teleoperation system, taking into account time-varying communication delay.

The Lyapunov Krasovskii functional is used to analyze delay-dependent stability and derive the stability criteria. The stability is guaranteed by choosing suitable control gains such that specific stability conditions are satisfied.

The proposed scheme also guarantees that the remote manipulator tracks the delayed local manipulator trajectory.

Finally, the simulation is presented to show the effectiveness of the main results.

Local-remote experimental test bed is currently under way and will be reported in the near future.

\section{ACKNOWLEDGMENT}

The authors are thankful for the financial support of Spanish Government CICYT Project Ref. DPI2009-08778 and also Comunidad de Madrid who support the project ROBOCITY2030-II Ref. P2009/DPI-1559 and for .the use of the facilities of the CETAM at the Pontificia Universidad Católica del Perú.

\section{REFERENCES}

[1] Goertz, R.C. and Thompson, R.C. : "Electronically controlled manipulator", Nucleonics, 1954, pp. 46-47

[2] Hannaford B. : "Stability and performance tradeoffs in bi-lateral telemanipulation". In Proc. IEEE Int. Conf. Rob. \& Auto, Scottsdale, AZ, May 1989, pp. 1764-1767

[3] Hokayem P.F.and Spong M.W. : "Bilateral teleoperation: An Historical survey", Automatica, 2006

[4] J.M Azorin, O. Reinoso, J.M. Sabater, R.P. Neco, and R. Aracil. "Dynamic analysis for a teleoperation system with time delay". In IEEE Conference on Control Applications, Istanbul, Turkey, volume 2, pages $1170-1175,2003$.

[5] Ganjefar et al., "Teleoperation systems design using augmented wavevariables and smith predictor method for reducing time-delay effects," in Proc. IEEE Int. Symp. Intelligent Control, Oct. 2002, pp. 333-338.

[6] Ferrell,W. R. : "Remote manipulation with transmission delay". IEEE Transactions on Human Factors in Electronics, 1965, 6, 1, pp. 24-32
[7] Anderson R. J., Spong M. W. : "Bilateral Control of Teleoperators with Time Delay". IEEE Transactions on Automatic Control, 1989, 34, (5), pp. 494-501

[8] Kim V.S, Hannaford B., and Bejczy A.K. : "Force-reflection and shared compliant control in operating telemanipulators with time delay". IEEE Trans, Rob. \& Auto, 1962, 8, (2), pp. 176-185

[9] Ferrel W.R. : "Delayed force feedback", IEEE Trans. Human Factors In Electronics, 1966, HFE8:449-455

[10] Melchiorri C. and Eusebi A. : "Telemanipulation: System aspects and control issues". Proc. Int. Summer School in Modelling and Control of Mechanisms and Robots, World Scientific, Bertinoro, Italy, 1996, pp. 149-183

[11] Yokokohji Y. and Yoshikawa T. : "Bilateral control of master-slave manipulators for ideal kinesthetic coupling-Formulation and experiment", IEEE Trans. Robot. Autom, 1994, 10, (5), pp. 605-620

[12] Lozano R., Chopra N. and Spong M. : "Convergence Analysis of Bilateral Teleoperation with Constant Human Input". In Proceedings of American Control Conference, 2007, pp. 1443-1448

[13] Nikhil Chopra and Mark W. Spong. "Delay-Independent Stability for Interconnected Nonlinear Systems with Finite L2 Gain". Proceedings of the 46th IEEE Conference on Decision and Control. New Orleans, LA, USA, Dec. 12-14, 2007.

[14] Emmanuel Nuño, Romeo Ortega, Nikita Barabanov, and Luis Basañez. A "Globally Stable PD Controller for Bilateral Teleoperators". IEEE TRANSACTIONS ON ROBOTICS, VOL. 24, NO. 3, JUNE 2008.

[15] Azorin J M., Reinoso O., Aracil R and Ferre M. : "Generalized control method by state convergence for teleoperation systems with time delay". Automatica, 2004b, 40, pp. $1575-1582$

[16] Azorin, J.M., Reinoso, O., Aracil, R, Ferre, M. : "Control of teleoperators with communication time delay through state convergence", Journal of Robotic Systems, 2004a, 21 (4), pp. 167-182

[17] Peña C. Trabajo Tesis Doctoral. "Control Bilateral por Convergencia de Estados para Sistemas Teleoperados con Robots de Cinemática Diferente". Universidad Politécnica de Madrid. España, 2009.

[18] Artigas, M., Aracil, R., Ferre, M., Garcia, C. : "Adaptive bilateral control systems through state convergence in teleoperation", RIAI Revista Iberoamericana de Automatica e Informatica Industrial, 2010, 7, (3), pp. $42-50$

[19] Tafur J.C., Garcia C., Aracil R \& Saltaren R. "Control of nonlinear teleoperation system by state convergence", Control and Automation (ICCA), 2011 9th IEEE International Conference on, Santiago de Chile, Chile, Page(s): 489 - 494, 2011.

[20] Kelly R. and Santibáñez V. : Control de Movimiento de Robots Manipuladores. Prentice Hall 2003

[21] Gu K., Niculescu S. (2003). Survey on Recents Results in the Stability and Control of Time-Delayed Systems. Transactions of the ASME, Vol. 125, June.

[22] Xu S. and Lam J. (2005). Improved Delay-Dependent Stability Criteria for Time-Delay Systems. IEEE Trans. On Automatic Control, Vol. 50, No. 3, March.

[23] Papachristodoulou A., Peet M, Lall S. (2005). Constructing LyapunovKrasovskii Functionals For Linear Time Delay Systems. American Control Conference, June 8-10, USA.

[24] Slotine and Li (1991). Applied Nonlinear Control. Prentice Hall.

[25] Teel A. R. (1999). Asymptotic convergence from Lp stability. IEEE Transaction on Automatic Control, 44(11):2169 - 2170 Nov.

[26] Hassan K. Khalil. Nonlinear Systems. Prentice Hall, NJ, third edit edition, 2002. ISBN 0-13-067389-7.

[27] Tafur J.C., Peña C, Aracil R. \& Garcia C. : "An Implemented of a Real-Time Experimental Setup for Robotic Teleoperation System". In Proceedings of the 11th International Workshop on Research and Education in Mechatronics - REM 2010, Ostrava, Czech Republic, pp. 41-48, September 2010.

[28] Oboe R., Fiorini P , "A Design and Control Environment for InternetBased Telerobotics". The International Journal of Robotics Research, 1998.

[29] Delgado E. , Díaz-Cacho M, Bustelo D, Barreiro A. Stability of teleoperation systems under time-varying delays by using LyapunovKrasovskii techniques. Proceedings of the 18th IFAC World Congress Milano (Italy) August 28 - September 2, 2011. 